\title{
Biomechanics and clinical experience of a 3D biomimicking vascular stent
}

Wolfram Schmidt, Institute for Biomedical Engineering, Rostock University Medical Center, FriedrichBarnewitz-Str. 4, 18119 Rostock-Warnemünde, Germany

Christian Wissgott, Institute of Diagnostic and Interventional Radiology / Neuroradiology, Westkuestenklinikum Heide, Academic Teaching Hospital of the Universities of Kiel, Luebeck and Hamburg, Esmarchstraße 50, 25746 Heide, Germany

Christoph Brandt-Wunderlich, Institute for ImplantTechnology, Friedrich-Barnewitz-Str. 4, 18119 RostockWarnemünde, Germany

Peter Behrens, Institute for Biomedical Engineering, Rostock University Medical Center, Friedrich-BarnewitzStr. 4, 18119 Rostock-Warnemünde, Germany

Klaus-Peter Schmitz, Institute for ImplantTechnology and Biomaterials and Institute for Biomedical Engineering, Rostock University Medical Center, Friedrich-Barnewitz-Str. 4, 18119 Rostock-Warnemünde, Germany

Niels Grabow, Institute for Biomedical Engineering, Rostock University Medical Center, Friedrich-BarnewitzStr. 4, 18119 Rostock-Warnemünde, Germany

Reimer Andresen, Institute of Diagnostic and Interventional Radiology / Neuroradiology, Westkuestenklinikum Heide, Academic Teaching Hospital of the Universities of Kiel, Luebeck and Hamburg, Esmarchstraße 50, 25746 Heide, Germany

The presented investigation was designed to assess the biomechanical behavior of a 3D biomimicking selfexpanding stent with respect to general technical parameters. First clinical experiences will be presented based on stent implantation and 24 months follow up.

Biomimics 3D stents (Veryan Medical) with $6.0 \mathrm{~mm}$ expanded diameter and $80 \mathrm{~mm}$ length $(\mathrm{n}=2)$ were used for biomechanical investigations. As parameters, the profile of the delivery catheter with mounted stent, the strut thickness, the bending stiffness, the axial stiffness for tension and compression and the length change during expansion were measured. Surface coverage as the measure of metal-to-artery ratio was determined using an indirect method.

The bending stiffness at small deflection was measured with $2.73 \mathrm{Nmm}^{2}$, the axial stiffness at tension was $7.77 \mathrm{~N} / \mathrm{mm}$ and at compression $97.61 \mathrm{~N} / \mathrm{mm}$. The stent shortened during expansion by $11.54 \%$. The radial force at $5 \mathrm{~mm}$ diameter was $2.54 \mathrm{~N}$. In the clinical test the stent was easy and save to deploy. Accurate placement was achieved by slow deployment. The implantation was technical and clinical successful. There were no stent fractures observed after 24 months. Duplex sonography showed stent patency without any binary restenosis or intimal hyperplasia.

The measured mechanical parameters were comparable to established SFA stents. The stent is highly flexible at bending and exerts a low radial force to the vasculature. The length shortening during expansion demands cautious deployment for accurate placement. The strut thickness of about $230 \mu \mathrm{m}$ is relatively high even for SFA stents. The axial stiffness for tension and compression is low which is assumed to adequately match to the vasculature. The helical structure is unique. It appears that the helical stent structure has an improved long-term patency. 


\section{Novel 3D printing concept for the fabrication of time-controlled drug delivery systems}

Jan Konasch, Fluid Technology and Microfluidics, University of Rostock, Rostock, Germany, jan.konasch@unirostock.de

Alexander Riess/ Robert Mau / Hermann Seitz, Fluid Technology and Microfluidics, University of Rostock, Rostock, Germany, alexander.riess@ uni-rostock.de/ robert.mau@uni-rostock.de/ hermann.seitz@uni-rostock.de

Michael Teske/ Natalia Rekowska/ Thomas Eickner/ Niels Grabow, Institute for Biomedical Engineering, University Medical Center Rostock, Rostock, Germany, michael.teske@uni-rostock.de/ natalia.rekowska@uni-rostock.de/ thomas.eickner@uni-rostock.de/niels.grabow@uni-rostock.de

Three-dimensional (3D) printing has become a popular technique in many areas. One emerging field is the use of 3D printing for the development of 3D drug delivery systems (DDS) and drug-loaded medical devices. A novel fabrication concept for the fabrication of time-controlled drug delivery systems based on stereolithography combined with inkjet printing is presented. An inkjet printhead and an UV-LED light source have been integrated into an existing stereolithography system. Inkjet printing is used to selectively incorporate active pharmaceutical ingredients (API) during a stereolithographic 3D printing process. In an initial experimental study, poly (ethylene glycol) diacrylate (PEGDA) was used as polymer whereas 2-Hydroxy-4'-(2-hydroxyethoxy)-2-methylpropiophenone (Irgacure 2959) and Lithium phenyl-2,4,6-trimethylbenzoylphosphinate (LAP) were used as photoinitiators. Basic structures could be manufactured successfuly by the new hybrid 3D printing system. 


\section{Histological evaluation of a cochlear implant electrode array with electrically activated shape change for perimodiolar positioning}

Thomas S. Rau, Department of Otolaryngology and Cluster of Excellence EXC 1077/1 “Hearing4all”, Hannover Medical School, Carl-Neuberg-Str. 1, 30625 Hannover, Germany, rau.thomas@mh-hannover.de

Nuha Suzaly, Department of Otolaryngology and Cluster of Excellence EXC 1077/1 “Hearing4all”, Hannover Medical School, Carl-Neuberg-Str. 1, 30625 Hannover, Germany, Suzaly.nuha@mh-hannover.de

Nick Pawsey, Cochlear Limited, 1 University Avenue, Macquarie University, Sydney, NSW 2109, Australia, NPawsey@cochlear.com

Silke Hügl, Department of Otolaryngology and Cluster of Excellence EXC 1077/1 “Hearing4all”, Hannover Medical School, Carl-Neuberg-Str. 1, 30625 Hannover, Germany, huegl.silke@mh-hannover.de

Thomas Lenarz, Department of Otolaryngology and Cluster of Excellence EXC 1077/1 “Hearing4all”, Hannover Medical School, Carl-Neuberg-Str. 1, 30625 Hannover, Germany, Lenarz.thomas@mh-hannover.de

Omid Majdani, Department of Otolaryngology and Cluster of Excellence EXC 1077/1 “Hearing4all”, Hannover Medical School, Carl-Neuberg-Str. 1, 30625 Hannover, Germany, Majdani.omid@mh-hannover.de

For the treatment of deafness or severe hearing loss cochlear implants (CI) are used to stimulate the auditory nerve of the inner ear. In order to produce an electrode array which is both atraumatic and reaches a perimodiolar final position a design featuring shape memory effect was proposed. A Nitinol wire with a diameter of $100 \mu \mathrm{m}$ was integrated in a state of the art lateral wall electrode array. The wire serves as an actuator after it has been 'trained' to adopt the spiral shape of an average human cochlea. Three small diameter platinum-iridium wires (each $20 \mu \mathrm{m}$ ) were crimped to the Nitinol wire in order to produce thermal energy. An insertion test was pursued using a human temporal bone specimen. The prototype electrode array was cooled down by means of immersion in ice water and freeze spray to enable sufficient straightening. Thereafter, insertion into the cochlea through the round window as performed. Insertion was feasible but difficult as premature curling of the electrode occurred during the movement towards the inner ear while passing the middle ear cavity. Therefore, the insertion had to be performed faster than usual. The shape memory actuator was subsequently activated with $450 \mathrm{~mA}$ current at $5 \mathrm{~V}$ for 3 seconds. After insertion the specimen was embedded in epoxy resin, microgrinded and all histological slices were assessed for trauma. Perimodiolar position was achieved. No insertion trauma was observed and there were no indications of thermal damage caused by the electrical heating. To the best of our knowledge, this is the first histological evaluation of the insertion trauma caused by an electrically activated shape memory electrode array. These promising results support further research on shape memory CI electrode arrays. 


\section{Hemodynamic influence of design parameters of novel venous valve prostheses}

Michael Stiehm, Institute for ImplantTechnology and Biomaterials e.V., Friedrich-Barnewitz-Str. 4, 18119 RostockWarnemünde, Germany, e-mail: michael.stiehm@uni-rostock.de

Stefanie Kohse, Institute for Biomedical Engineering, Rostock University Medical Center, Friedrich-Barnewitz-Str. 4, 18119 Rostock-Warnemünde, Germany, e-mail: stefanie.kohse@uni-rostock.de

Kerstin Schümann, Institute for Biomedical Engineering, Rostock University Medical Center, Friedrich-Barnewitz-Str. 4, 18119 Rostock-Warnemünde, Germany, e-mail: kerstin.schuemann@uni-rostock.de

Sebastian Kaule, Institute for ImplantTechnology and Biomaterials e.V., Friedrich-Barnewitz-Str. 4, 18119 RostockWarnemünde, Germany, e-mail: sebastian.kaule@uni-rostock.de

Stefan Siewert, Institute for ImplantTechnology and Biomaterials e.V., Friedrich-Barnewitz-Str. 4, 18119 RostockWarnemünde, Germany, e-mail: stefan.siewert@uni-rostock.de

Jan Oldenburg, Institute for Biomedical Engineering, Rostock University Medical Center, Friedrich-Barnewitz-Str. 4, 18119 Rostock-Warnemünde, Germany, e-mail: jan.oldenburg@uni-rostock.de

Jonas Keiler, Institute of Anatomy, Rostock University Medical Center, Getrudenstr. 9, 18057 Rostock, e-mail: Jonas.keiler@uni-rostock.de

Niels Grabow, Institute for Biomedical Engineering, Rostock University Medical Center, Friedrich-Barnewitz-Str. 4, 18119 Rostock-Warnemünde, Germany, e-mail: niels.grabow@uni-rostock.de

Andreas Wree, Institute of Anatomy, Rostock University Medical Center, Getrudenstr. 9, 18057 Rostock Germany, email: andreas.wree@uni-rostock.de

Klaus-Peter Schmitz, Institute for ImplantTechnology and Biomaterials e.V., Friedrich-Barnewitz-Str. 4, 18119 Rostock-Warnemünde, Germany, e-mail: klaus-peter.schmitz@uni-rostock.de

Venous ulcers of lower limbs are one clinical manifestation of chronic venous insufficiency. Currently, there is no venous valve prosthesis available. This study presents novel venous valve prostheses made of three-dimensional electrospun fibrous nonwovens leaflets. The aim of this study was to prove the feasibility of the manufacturing process as well as to investigate design features of the venous valve prostheses from a hemodynamic point of view. An adapted pulse duplicator system (ViVitrolabs, Victoria, BC, CA) was used for characterization of the hydrodynamic performance. For eight different venous valve prototypes flow rate, effective orifice area and regurgitation fraction was investigated in vitro. In particular, tricusp valve designs showed an up to $40 \%$ higher effective orifice area as well as $15 \%$ higher maximum flowrate compared to bicusp valve designs. However, the regurgitation fraction of the bicusp valve designs is up to $86 \%$ lower compared to tricusp valve. Additionally, the hemodynamic performance of the tricuspid valves showed a high sensitivity regarding the leaflet length. Bicuspid valves are less sensitive to changes of design parameters, more sufficient and therefore highly reliable. 


\section{Development and validation of a tissue-equivalent test environment for detection of malfunctions in active medical implants caused by ionizing radiation}

Manuel Stich ${ }^{1,2 *}$, Larissa Blümlein ${ }^{1,3}$, Anne Slawig ${ }^{1,2}$, Felix Schmidl ${ }^{1}$, Karina Schuller ${ }^{1}$, Richard Lösch $^{3}$, Matthias Hipp ${ }^{3}$, Sabine Hentschel ${ }^{3}$, Gregor Schaefers ${ }^{4}$ and Ralf Ringler ${ }^{1}$

* First and corresponding author: m.stich@oth-aw.de

${ }^{1}$ X-Ray and Molecular Imaging Lab, OTH - Technical University of Applied Sciences AmbergWeiden, Hetzenrichter Weg 15, Weiden (92637), Germany

${ }^{2}$ Institute for Diagnostic and Interventional Radiology, University of Würzburg, Oberdürrbacher Str. 6, Würzburg (97080), Germany

${ }^{3}$ Department of Radiation Oncology, Hospital St. Marien Amberg, Mariahilfbergweg 7, Amberg (92224), Germany

\section{${ }^{4}$ MR:comp GmbH, 45892 Gelsenkirchen, Germany}

Many patients in radiotherapy carry active implantable medical devices (AIMDs) such as pacemakers (ICPs) or defibrillators (ICDs). The influence of the ionizing radiation can lead to failures in the device function. This study presents a tissue-equivalent test environment to investigate the influence of ionizing radiation on AIMDs in a realistic and reproducible test setup.

The in-vitro test environment is designed to simulate a human torso. Structures such as the heart, lungs, ribs, spinal column and soft tissue are replicated from tissue-equivalent materials to allow realistic treatment planning and to simulate the effect of ionizing radiation on active implants. Numerous materials such as polymers, resins or gels were tested for their suitability as a substitute, using CT scans for selection and Monte-Carlo simulations and dose measurements with a two-dimension ionization chamber array for final material property validation. The phantom should be applicable in the full treatment chain which means that the treatment planning process should be feasible as with a patient and the planned and applied dose have to be comparable.

It can be shown that Polytetrafluorethylen (bone), carrageenan (heart), Styrodur (lung) and Biresin®G27 (soft tissue) fulfill all requirements for suitable tissue surrogates. The respective organs were formed from the surrogate materials and combined into an anthropomorphic phantom of the upper body. A plug-in unit integrated in the test environment has been designed specifically to allow the placement of electronic components and AIMDs in the phantom at typical positions for implant placement in humans. For dose verification, dose measurement devices like ionization chambers can be integrated near the AIMDs and at different positions next to organs using the plug-in unit. The test environment is applicable in the full treatment planning process in radiation therapy with X6 photons. The planned dose can be verified using dose measurements with an accuracy of $\sim 2 \%$. 


\section{Signal Processing in Medical Implants}

Uwe Marschner: Institute of Semiconductors and Microsystems, Technische Universität Dresden, D-01062 Dresden, Germany, e-mail: uwe.marschner@tu-dresden.de

Wolf-Joachim Fischer: Chair of Microsystems, Technische Universität Dresden, D-01062 Dresden

Wilfried Mokwa: RWTH Aachen, Department of Materials in Electrical Engineering 1, Aachen, Germany

Thomas Stieglitz: Laboratory for Biomedical Microtechnology

Department of Microsystems Engineering, University of Freiburg - IMTEK, Freiburg, Germany.

Intelligent or sensory functional implants are - from an elec-trical viewpoint - highly complex systems of sensors, actua-tors and signal processing. They can measure and evaluate intracorporeal quantities permanently and are used in in vivo diagnostics, in vivo monitoring and in therapy. Prerequisite for an autonomous therapy by the implant itself is a suitable signal analysis for the diagnosis. Current sensory functional implants draw their energy from a battery or an extracorpo-real electromagnetic field. Current developments in the field of energy harvesting, while significantly reducing the power consumption of the electronic circuits, let expect a trend towards long-term stable autonomous, theranostic implants with complex signal processing. 